\title{
The Role of Interprofessional Education in the Development of Healthcare Professionals' Team Skills
}

\author{
Katie Sniffen \\ Saint Louis University, katie.sniffen@health.slu.edu \\ Noor Al-Hammadi \\ Saint Louis University, noor.alhammadi@health.slu.edu \\ Leslie Hinyard \\ Saint Louis University, leslie.hinyard@health.slu.edu
}

Follow this and additional works at: https://nsuworks.nova.edu/ijahsp

Part of the Interprofessional Education Commons

\section{Recommended Citation}

Sniffen K, Al-Hammadi N, Hinyard L. The Role of Interprofessional Education in the Development of Healthcare Professionals' Team Skills. The Internet Journal of Allied Health Sciences and Practice. 2021 Jan 01;19(3), Article 10.

This Manuscript is brought to you for free and open access by the College of Health Care Sciences at NSUWorks. It has been accepted for inclusion in Internet Journal of Allied Health Sciences and Practice by an authorized editor of NSUWorks. For more information, please contact nsuworks@nova.edu. 


\title{
The Role of Interprofessional Education in the Development of Healthcare Professionals' Team Skills
}

\begin{abstract}
Purpose: Interprofessional education (IPE) across the health professions learning continuum is suggested to prepare health professionals for interprofessional collaborative practice (IPCP) through development of necessary attitudes, knowledge, behaviors, and skills. The purpose of this study was to investigate the flow-on effects of previous IPE experiences across the learning continuum on existing IPCP skills and behaviors in healthcare professionals. Methods: A retrospective observational study design was used to survey a convenience sample of healthcare professionals working in IPCP clinics about their selfreported team and collaboration skills via the pre-existing Team Skills Scale (TSS) and Self-Assessed Collaborative Skills (SACS) tools. Additional survey items were created by the research team based on self-reported prior IPE exposure, timing of initial IPE exposure, and number of IPE exposures. Sixty-three participants completed the survey ( $28 \%$ response rate). Results: No statistically significant differences were found between groups across all categories of IPE exposure. Non-significant trends suggest higher team and collaboration skills are related to earlier and cumulative IPE exposures throughout learning and professional development. Conclusion: IPE as a broad definition is not sufficient to detect significant relationships with healthcare professionals' IPCP skills. Future studies should investigate the longitudinal effectiveness of specific IPE interventions on distal IPCP skills and account for other contributing factors.
\end{abstract}

\section{Author Bio(s)}

Katie Sniffen, MS, ATC, is the Program Coordinator for Saint Louis University's AHEAD Institute and an Instructor in SLU's Athletic Training Program with additional teaching experience in Interprofessional Education, Katie is also pursuing a PhD in Health Outcomes Research.

Noor Al-Hammadi, MPH, is a senior biostatistician at Saint Louis University's AHEAD Institute. She received her MPH in Epidemiology and Biostatistics and is currently in training for a PhD in Health Outcomes Research. Noor has over 10 years of experience working as a biostatistician on several $\mathrm{NIH}$-funded grants.

Leslie Hinyard, PhD, MSW is Chair and Associate Professor in the Department of Health and Clinical Outcomes Research and the founding Director of the AHEAD Institute at Saint Louis University.

\section{Acknowledgements}

The authors would like to thank the Center for Interprofessional Education and Research (CIER) for their support in this study and the Site Coordinators of the clinics in partnership with the CIER for their help distributing the e-survey. This research did not receive any specific grant from funding agencies in the public, commercial or not-for-profit sectors. 


\title{
IIIAHSP \\ The Internet Journal of Allied Health Sciences and Practice \\ Dedicated to allied health professional practice and education \\ Vol. 19 No. 3 ISSN 1540-580X
}

\section{The Role of Interprofessional Education in the Development of Healthcare Professionals' Team Skills}

\author{
Katie Sniffen \\ Noor Al-Hammadi \\ Leslie Hinyard \\ St. Louis University \\ United States
}

\begin{abstract}
Purpose: Interprofessional education (IPE) across the health professions learning continuum is suggested to prepare health professionals for interprofessional collaborative practice (IPCP) through development of necessary attitudes, knowledge, behaviors, and skills. The purpose of this study was to investigate the flow-on effects of previous IPE experiences across the learning continuum on existing IPCP skills and behaviors in healthcare professionals. Methods: A retrospective observational study design was used to survey a convenience sample of healthcare professionals working in IPCP clinics about their self-reported team and collaboration skills via the pre-existing Team Skills Scale (TSS) and Self-Assessed Collaborative Skills (SACS) tools. Additional survey items were created by the research team based on self-reported prior IPE exposure, timing of initial IPE exposure, and number of IPE exposures. Sixty-three participants completed the survey (28\% response rate). Results: No statistically significant differences were found between groups across all categories of IPE exposure. Non-significant trends suggest higher team and collaboration skills are related to earlier and cumulative IPE exposures throughout learning and professional development. Conclusion: IPE as a broad definition is not sufficient to detect significant relationships with healthcare professionals' IPCP skills. Future studies should investigate the longitudinal effectiveness of specific IPE interventions on distal IPCP skills and account for other contributing factors.
\end{abstract}

Keywords: interprofessional relations, interdisciplinary communication, delivery of health care, education, patient care team 


\section{INTRODUCTION}

Complex and chronic health conditions affect patients across the entire lifespan. Sixty percent of adults in the United States (US) have at least one chronic condition and $40 \%$ suffer from multiple chronic conditions. ${ }^{1}$ Conditions such as hypertension, lipid disorders, mood disorders, and diabetes mellitus individually affect $10-27 \%$ of US adults. ${ }^{1}$ The number of chronic conditions experienced by a single person is associated with increased healthcare service utilization and healthcare spending. Individuals with five or more chronic conditions average 20 outpatient visits and 51 prescriptions per year. ${ }^{1}$

Effective management of complex and chronic conditions requires an interprofessional collaborative approach to ensure a patientcentered model of health care delivery. 2,3 Interprofessional collaborative practice (IPCP) takes place when "multiple health workers from different professional backgrounds provide comprehensive services by working with patients, their families, caregivers, and

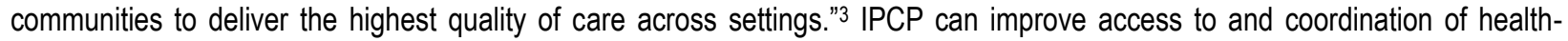
services, appropriate use of specialist clinical resources, health outcomes for people with chronic diseases, and patient safety. ${ }^{4-6}$ IPCP can also decrease total patient complications, length of hospital stay, tension and conflict among caregivers, staff turnover, hospital admissions, clinical error rates, and mortality rates. $.7-74$

The World Health Organization has placed a call to action for intentionally preparing the healthcare workforce for IPCP through the inclusion of interprofessional education (IPE) in health professions training programs. ${ }^{3}$ Ensuring that IPE learning outcomes translate into professional practice behaviors is imperative to the successful preparation of collaboration-ready healthcare professionals. In this effort, investigating the distal impact of IPE exposure during training programs on IPCP skills in practicing healthcare professionals could offer insights into the longitudinal effects of IPE.

\section{BACKGROUND}

\section{Interprofessional Education}

IPE is defined as experiences where "two or more professions learn about, from and with each other to enable effective collaboration and improve health outcomes." ${ }^{3} \mathrm{It}$ is important to note that IPE requires a formal and intentional curricular design that includes critical reflection on collaboration skills such as roles and responsibilities, effective communication, and teamwork processes..$^{15}$ The Institute of Medicine (IOM) describes the learning continuum of IPE experiences across the health professions training trajectory in their Framework for Measuring the Impact of IPE. ${ }^{16}$

\section{Learning Continuum}

According to the IOM Framework, IPE can occur at three distinct time points across health professions education and training. Foundational education encompasses the learning of novice individuals, usually prior to training of profession-specific skills, and includes underlying competencies of teamwork and collaboration. These experiences typically occur in the classroom and have been shown to result in positive learning outcomes related to attitudes, perceptions and knowledge of IPCP. ${ }^{16}$ Graduate education, defined by the IOM Framework as "any advanced formal or supervise health professions training taking place between completion of foundation education and entry into unsupervised practice," introduces more complex IPE, often in the form of interprofessional case-based simulations. Competencies and learning outcomes of these experiences are typically informed by profession-specific accreditation standards and therefore can vary considerably between professions. ${ }^{16}$ For practicing healthcare professionals, continuing professional development can include IPE to promote the skills necessary for collaborative systems of care. ${ }^{16}$ With a system-wide shift towards the creation of "learning organizations," these IPE experiences can take the form of formal continuing education or less-formal workplace activities. ${ }^{17}$ The Health Professions Accreditors Collaborative (HPAC) articulates that the longitudinal nature of these experiences is necessary to successfully achieve the learning outcomes of IPE. ${ }^{18}$ Therefore, participating in IPE experiences at multiple timepoints across education, training, and practice may be important in developing IPCP skills.

\section{Enabling/Interfering Factors}

The Framework also highlights the enabling or interfering factors that could impact the success of IPCP achieving quality outcomes. These include professional culture, institutional culture, workforce policy, and financing \& reimbursement policies. Each profession has its own set of values, language, and norms curated during educational training while students establish their professional identity. ${ }^{19}$ Conflicting aspects of professional culture can interfere with an interprofessional team's ability to engage in shareddecision making, responsibility, and leadership. ${ }^{20} \mathrm{An}$ institution organized in professional silos fosters a culture that discourages collaboration. To enable interprofessional collaboration, there must be institutional support from leadership with sufficient time and space allocated for such interactions.,21 Workforce policy needs to support IPCP with clearly defined procedures for shared governance, shared decision-making, and regular communication. ${ }^{3}$ Additionally, financing and reimbursement policies that incentivize collaborative practice and their associated outcomes would foster greater motivation for actively engaging in teambased care. These factors will be important to account for when evaluating the demonstration of IPCP skills in a clinical setting. 


\section{Individual Factors}

Other individual factors may be important in the development of collaboration skills. Differences in competencies emphasized by profession-specific accreditation standards may result in differences in the skills developed by respective professionals during education and training. The cultural qualities of each profession will also influence an individual's attitudes, beliefs, and behaviors related to teamwork as they are socialized into their professional identity. ${ }^{20}$ Years of experience in practice or with the IPCP clinic will vary the exposures to different interprofessional experiences. Individual social and cultural factors associated with age, gender, race, and ethnicity may play an important role in one's ability to carry out certain skills. These relationships are ill-defined in the literature regarding their influence on the development and demonstration of IPCP skills.

\section{Measuring IPE}

Abundant research exists describing the changes of health professions student's attitudes towards IPCP after engaging in IPE.22 More emphasis needs to be placed on understanding the longitudinal translation of IPE to IPCP skills and behaviors. ${ }^{16}$ Informed by Kirkpatrick's model for evaluation, the IOM framework suggests moving beyond learning outcomes evaluation of attitudes and perceptions to the assessment of actual practice behaviors and skills. ${ }^{23}$ Limited studies have reported on skills and behavior outcomes. ${ }^{24}$ Even less have done the statistical analyses required to determine if a significant relationship exists between IPE and IPCP skills. ${ }^{22}$ As the inclusion of IPE experiences in health professions education programs continues to grow, graduates of these programs are integrating into the workforce. There is a need to evaluate the effect these learning experiences are having on the practice of interprofessional skills in the clinical workplace.

The objective of this study was to analyze the relationship between prior IPE experiences and self-rated IPCP skills among practicing healthcare professionals. In doing so, we addressed the following research aims:

Aim1: Determine if there is an association between any prior IPE exposure and health professionals' self-rated IPCP skills.

Aim 2: Determine if there is an association between the timing of initial exposure to IPE and health professionals' selfrated IPCP skills.

Aim 3: Determine if there is an association between the number of prior IPE exposures and health professionals' selfrated IPCP skills.

\section{METHODS \\ Study Design}

This study was a retrospective, cross-sectional, non-equivalent groups design using a convenience sample of a known network with a two-step approach to participant recruitment. This study was approved by the St. Louis University's Institutional Review Board.

\section{Participant Recruitment}

Participants were recruited from a cohort of 19 health care services clinics currently partnering with a university's Center for Interprofessional Education and Research (CIER). By limiting our sample to these clinics, we attempted to control for the potentially enabling or interfering professional and workplace variables as outlined in the IOM framework. Partnering clinics have been identified as positive examples of IPCP models for students to observe effective team-based care in practice during a series of clinical observation rotations. As identified models of IPCP, the underlying assumption is that sufficient enabling factors are in place at each clinical site to practice effective team-based care. The settings of these clinics included early childhood development centers, skilled nursing facilities, memory care units, inpatient and outpatient pediatric rehabilitation hospitals, hospice and palliative care services, cardiac rehabilitation clinics, family and community medicine clinics, ALS treatment centers, and surgical centers. Staff roles within each clinic include a variety of health professions, including physicians, nurse practitioners, nurses, physical therapists, occupational therapists, social workers, speech therapists, and dieticians. E-surveys were sent via email to the Clinical Site Coordinators for distribution to each clinic's team members. Clinical Site Coordinators were eligible to participate in the survey.

\section{Survey Development}

Survey participants were provided with an introduction designed to help participants identity if they had participated in IPE. IPE participation and timing of IPE served as the main predictor variables for this study. Definitions were reviewed for content validity by experts in the IPE field and health care providers not involved in the study. If respondents indicated prior participation in IPE, they were asked to specify when they participated in IPE:

- Foundational Education: IPE occurred during your bachelor's degree program (i.e college undergraduate major, associate's degree) 
- Graduate Education: IPE occurred during your graduate degree program (i.e., professional master's degree programs, medical school, clinical doctoral programs)

- Continuing Professional Development: IPE occurred during your licensed healthcare practice (i.e., continuing education credits, employer sponsored workshops)

Outcome variables included team skills and self-assessed collaboration. Team skills were assessed using the Team Skills Scale (TSS). The TSS has been used extensively as a self-perceived measure of team skills, initially in geriatric patient settings. ${ }^{25-29} \mathrm{It}$ requires responses to 17 items that capture the competencies for IPCP outlined by the Interprofessional Education Collaborative: values and ethics, roles and responsibilities, effective communication, and team processes and outcomes. ${ }^{30}$ Each question rates on a 5-point Likert scale that ranges from poor (1) to excellent (5) with higher cumulative scores indicating better team skill levels. One statement in the instrument specifically indicated geriatric patient care principles and was adapted to be inclusive of all patient care settings. Responses to all items are needed to compute the cumulative score. Response scores were summed and used as a continuous metric for analysis.

Collaboration was measured using the Self-Assessed Collaboration Skills (SACS) instrument. ${ }^{31}$ The SACS instrument is a threedimensional scale that was developed and validated by research at Saint Louis University to measure collaboration skills in both academic and clinical settings. ${ }^{31}$ This scale covers competencies related to information sharing, team support, and learning. It is composed of 11 items, with a range of two-to-six questions per domain. Questions rate on a 7-point scale with answers ranging from strongly disagree (1) to strongly agree (7). Responses to all items are needed to compute the cumulative score. Response scores were summed and used as a continuous metric for analysis.

Other potential confounders and covariates included profession, years of experience, years with the IPCP clinic, age, gender, ethnicity, and race. Finally, participants were asked to indicate and comment on what they believed to be the contributing factors to their IPCP skills. This was a multiple response question that offered several options and included an open-ended "other" option for participants to provide their own response. This question was included to provide participant-reported insights to the contributing factors of IPCP skills that the research team may not have accounted for as covariates.

\section{Survey Administration}

The survey was administered using the Qualtrics Survey tool. Participants received an email containing the survey link from their Clinical Site Coordinator in March 2019. Participants were asked to respond within two weeks. Participation in the e-survey was voluntary and a drawing for a $\$ 20$ Amazon gift card was offered as incentive to participate in the study. Contact information was collected via a second form that participants could optionally link to upon survey completion. Contact information was not able to be linked to original survey responses. Language confirmations regarding the anonymity of responses were provided in both the survey and the contact information form.

The anonymous responses were exported for data analysis at the conclusion of the two-week data collection period. Reminder emails were sent at one week and two days prior to the closing of the survey. No additional advertisement was used to motivate participation.

\section{Statistical Analysis}

Frequencies, percentages, means and standard deviations (or medians and IQRs as appropriate) were used to report descriptive statistics and characterize the sample. Assessment of the outcome score differences between groups that participated in IPE and those that did not were examined using the t-test with a significant level of $p=0.05$. ANOVA with Tukey's pairwise comparisons were used to identify the outcome score differences across the levels of the timing of initial exposure to IPE and the number of exposures to IPE. The intraclass correlation coefficient was computed to assess the correlation and agreement between the TSS and SACS scores. ${ }^{32}$ Boxplots and the Bland Altman plot of endpoints were used for visual illustration of the data. Analyses were performed using SAS 9.4 (SAS Institute Inc., Cary, NC, USA).

\section{RESULTS}

\section{Descriptive Statistics}

The survey was sent to at least 226 healthcare professionals working in IPCP clinics partnered with the CIER. $28 \%$ of those who were sent the email viewed the survey. Of those that opened the survey, $93 \%$ participated and $86 \%$ completed all necessary outcome measures (TSS and SACS), resulting in a final sample of 63 participants (Figure 1). Most of the participants were female $(55 / 63,87 \%)$, White $(56 / 63,88.9 \%)$, and Non-Hispanic (62/63, 98.4\%). The samples' mean age was 43 years old (SD: 11.6$)$ with a median of 14 years of professional experience (IQR 5-21) and a median of 5 years on their current IPCP team (IQR 2-11). The majority of respondents reported at least one exposure to IPE $(36 / 63,57.1 \%)$ with most stating that their initial IPE exposure was 
during their professional development (13/35, 37.1\%). Forty-eight percent (15/35) of those that had any exposure to IPE had their experiences over at least two time points across the learning continuum. Descriptive statistics of the sample, including average TSS and SACS scores across levels of each characteristic can be found in Table 1.

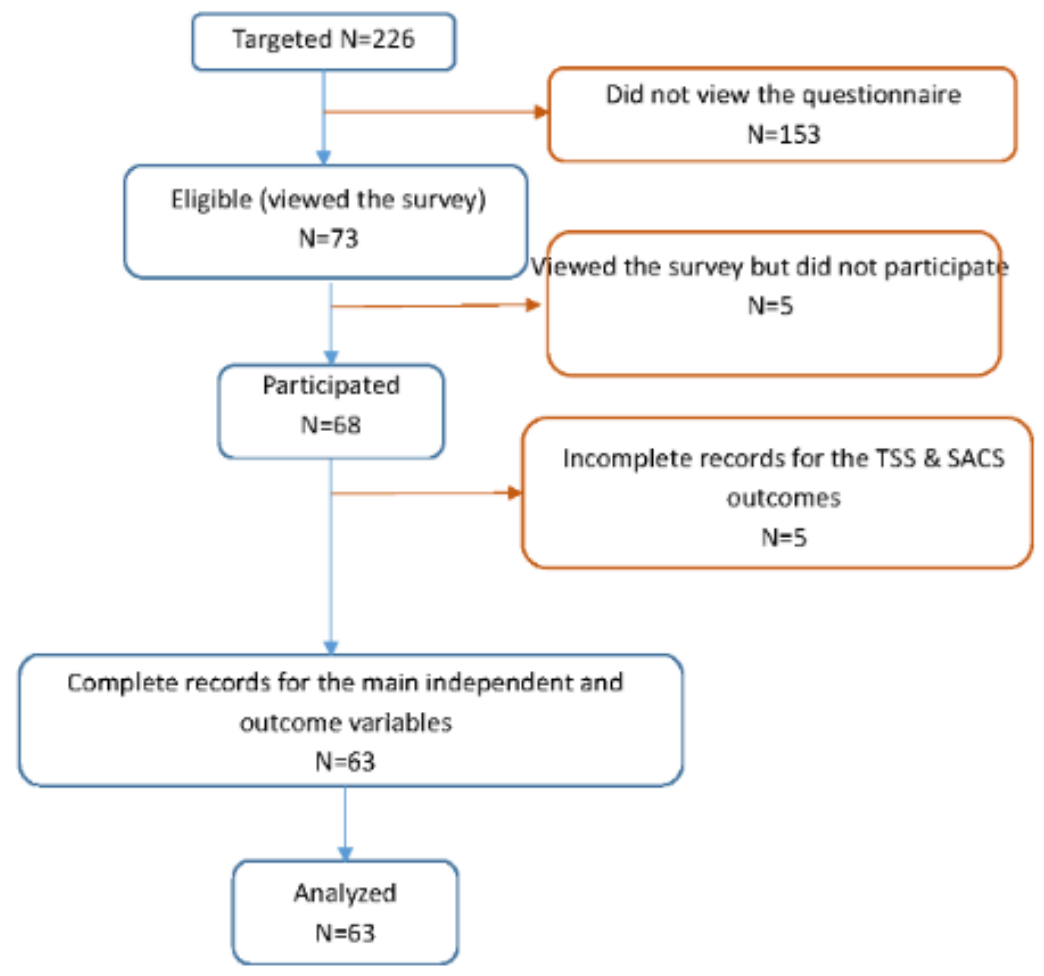

Figure 1. Consort diagram of survey recruitment and response

Table 1. Descriptive statistics of sample and average outcome scores

\begin{tabular}{|c|c|c|c|}
\hline Sample Characteristics & $n(\%)$ & $\begin{array}{c}\text { TSS } \\
\text { M (SD) }\end{array}$ & $\begin{array}{l}\text { SACS } \\
\text { M (SD) }\end{array}$ \\
\hline Age (M, SD) & $43.3(11.6)$ & & \\
\hline $21-35$ & $18(28.6)$ & $63.4(7.9)$ & $59.9(5.3)$ \\
\hline $36-50$ & $29(46.0)$ & $70.8(9.3)$ & $61.4(5.8)$ \\
\hline $51-70$ & $16(25.4)$ & $71.4(9.2)$ & $62.3(4.1)$ \\
\hline Years of Practice (Median, IQR) & $14(5,21)$ & & \\
\hline $1-10$ & $29(46.0)$ & $65.3(9.3)$ & $59.2(5.6)$ \\
\hline $11-20$ & $18(28.6)$ & $74.8(9.1)$ & $63.9(5.0)$ \\
\hline $21-30$ & $10(15.9)$ & $67.0(7.4)$ & $62.6(3.6)$ \\
\hline$>30$ & $6(9.5)$ & $71.7(4.7)$ & $60.7(2.7)$ \\
\hline Years on Team (Median, IQR) & $5(2,11)$ & & \\
\hline $1-10$ & $46(73.0)$ & $68.0(9.0)$ & $60.6(5.1)$ \\
\hline $11-20$ & $14(22.2)$ & $71.5(10.8)$ & $62.6(5.7)$ \\
\hline$>20$ & $3(4.8)$ & $69.3(9.6)$ & $64.0(6.1)$ \\
\hline \multicolumn{4}{|l|}{ Gender } \\
\hline Female & $55(87.3)$ & $69.0(11.9)$ & $61.0(3.2)$ \\
\hline Male & $6(9.5)$ & $68.7(9.1)$ & $61.2(5.5)$ \\
\hline \multicolumn{4}{|l|}{ Race } \\
\hline White & $56(88.9)$ & $69.2(9.3)$ & $61.3(5.3)$ \\
\hline Black & $3(4.8)$ & $60.3(9.7)$ & $58.7(6.0)$ \\
\hline Asian & $2(3.2)$ & $74.0(8.5)$ & $64.0(2.8)$ \\
\hline
\end{tabular}


Other

Profession

Behavioral Health Specialist

Dietician/Nutritionist

Nurse

Nurse Practitioner

Occupational Therapist

Pharmacist

Physical Therapist

Physician/MD

Speech Pathologist

Other-Administrative
$2(3.2)$

$4(6.3)$

$4(6.3)$

$10(15.9)$

$8(12.7)$

$5(7.9)$

$11(17.4)$

$2(3.2)$

$3(4.8)$

$4(6.3)$

$11(17.4)$
$67.0(11.3)$

$78.0(4.2)$

$79.3(8.3)$

$67.7(8.2)$

$68.4(7.8)$

$70.8(5.3)$

$67.7(12.1)$

$63.0(11.3)$

$62.7(3.8)$

$64.5(4.2)$

67.4 (11.3)
$59.5(4.9)$

$62.0(3.6)$

$64.8(4.5)$

$60.4(5.6)$

$61.8(6.9)$

$59.4(4.9)$

$61.4(7.4)$

$60.0(5.7)$

$61.3(5.9)$

$61.5(1.7)$

$60.8(4.2)$

\section{IPE Exposure}

Higher outcome scores were seen among those with any previous exposure to IPE (TSS: M=70, SD=9.6; SACS: M=61.7, $S D=5.4$ ) compared to participants with no exposure to IPE (TSS: $M=67.3, S D=9.1$; $S A C S: M=60.6, S D=5.1$ ). However, no significant differences in TSS or SACS sum scores were found between groups; TSS: $t(61)=-1.31, p=.26$; SACS: $t(61)=-.77$, $p=.44$ (Figure 3).

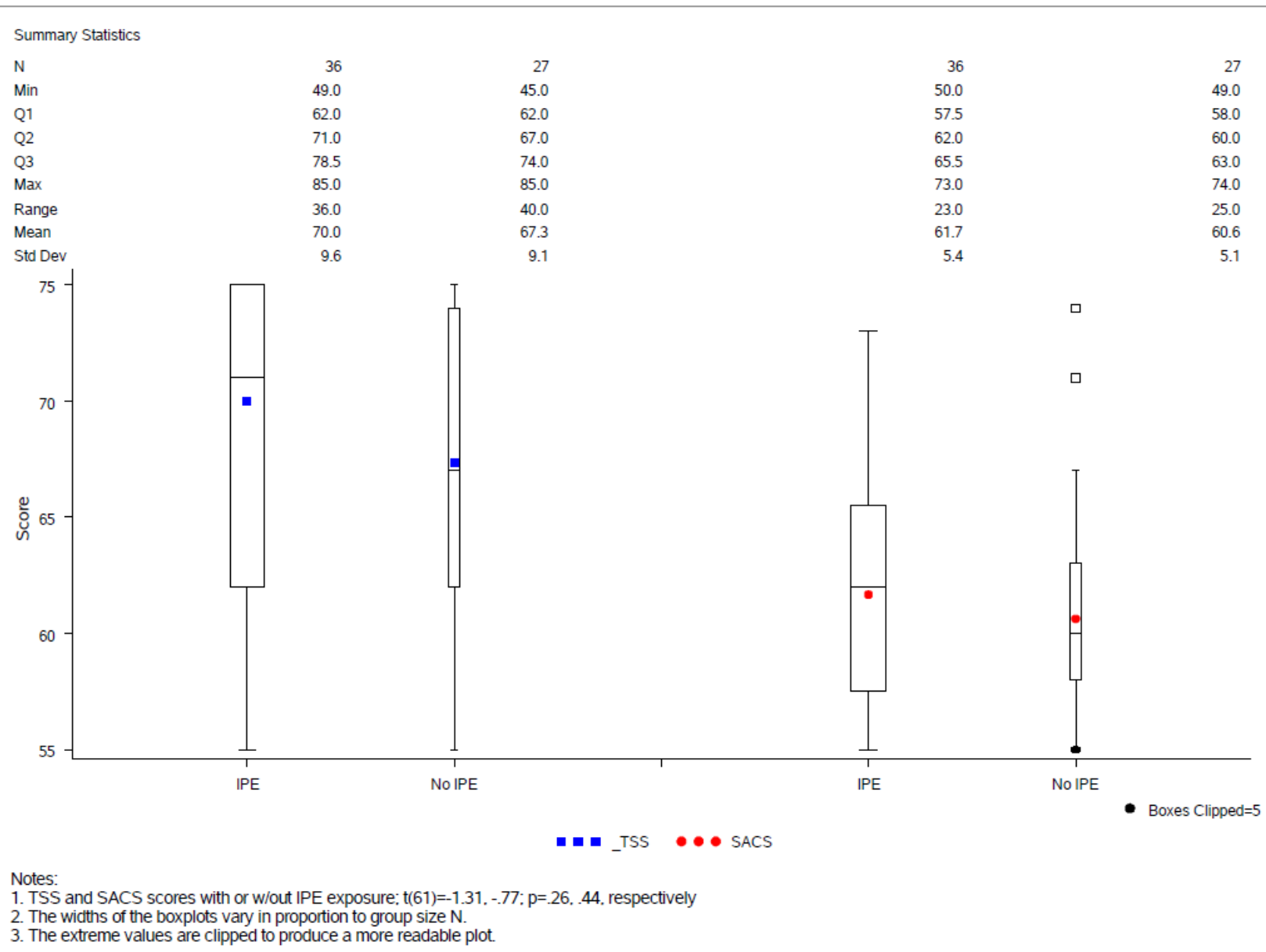

Figure 2. Boxplots of TSS and SACS scores with or without IPE exposure

\section{Timing of Initial IPE}

Higher outcome scores were seen among participants with initial IPE exposure during Foundational Education (TSS: M=72.8, $S D=10.0$; SACS: $M=63.6, S D=5.3$ ) than those with no IPE exposure (TSS: $M=66.7, S D=9.6 ; S A C S: M=60.8, S D=5.1$ ) or initial IPE exposure at the Graduate (TSS: $M=70.3, S D=8.5$; SACS: $M=62.6, S D=4.7$ ) or Professional levels (TSS: $M=69.1, S D=9.0$; 
SACS: $M=58.9, S D=5.4)$. However, no significant differences were seen among groups for either the TSS or SACS sum scores (TSS: $F(3,59)=1.25, p=.30 ; \operatorname{SACS}: F(3,59)=2.02, p=.12$ ) (Figure 4).

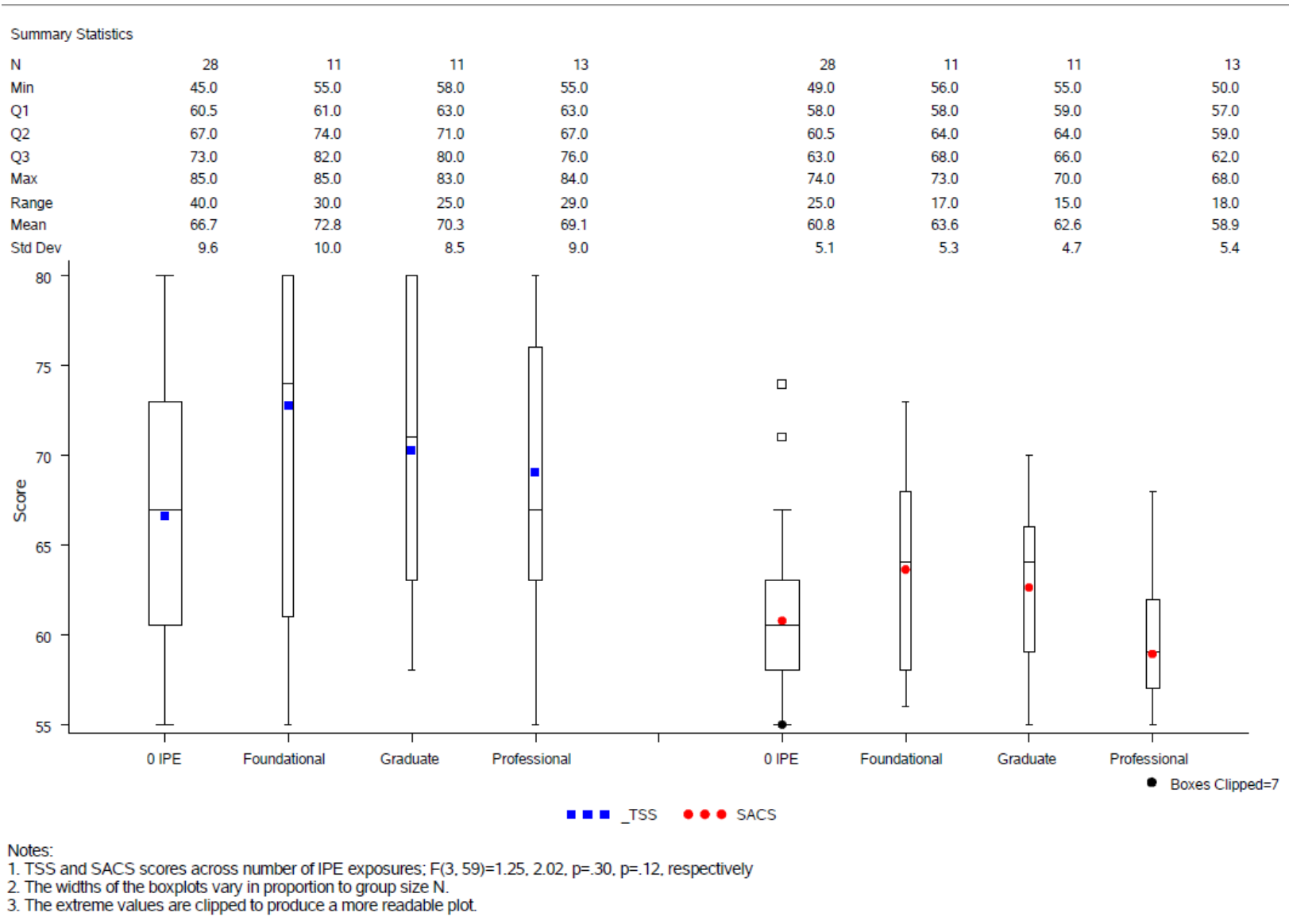

Figure 3. Boxplots of TSS and SACS scores across number of IPE exposures

\section{Number of IPE Exposures}

Higher outcome scores were seen for participants with two or more IPE exposures (TSS: M=71.4, SD=9.8; SACS: M=62.5, $\mathrm{SD}=5.0$ ) than one IPE exposure (TSS: $M=69.9, \mathrm{SD}=8.4$; $S A C S: M=60.7, \mathrm{SD}=5.8$ ) and no IPE exposure (TSS: $\mathrm{M}=66.7, \mathrm{SD}=9.6$; SACS: $M=60.8, S D=5.1)$. However, no significant differences were seen among groups for either the TSS or SACS sum scores; TSS: $F(2,60)=1.52, p=.23 ;$ SACS: $F(2,60)=.71, p=.49$ (Figure 5). 


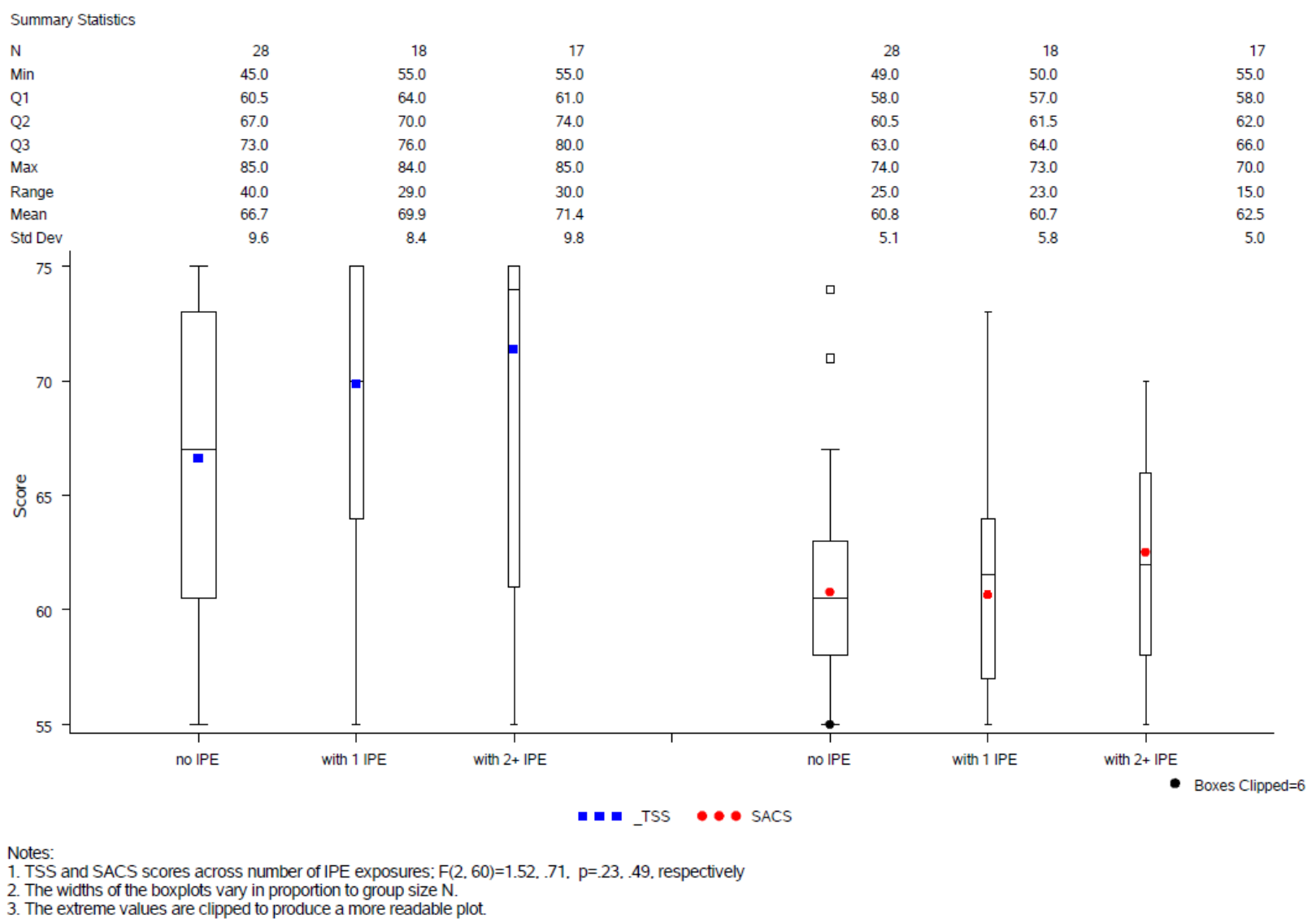

Figure 4. Boxplots of TSS and SACS scores across number of IPE exposures

\section{Self-Reported Contributing Factors}

Participants were given the opportunity to choose from multiple options indicating what they thought could have contributed to the development of their teamwork and collaboration skills. "Individual personal characteristics" was the most frequently selected response $(51 / 63,75 \%)$, followed by "previous work experience" (50/63, 73\%), "workplace culture" (37/63, 54\%) and "leadership support" (33/63, 48\%). Frequencies and percentages of each selection are presented in Table 2.

Table 2. Frequencies of self-reported contributing factors to development IPCP skills

\begin{tabular}{lr}
\hline Contributing Factor & $\mathbf{n}(\%)$ \\
\hline Individual personal characteristics & $51(75.0)$ \\
Previous work experiences & $50(73.5)$ \\
Workplace culture & $37(54.4)$ \\
Leadership support & $33(48.5)$ \\
Education experiences other than IPE & $24(35.3)$ \\
Mentorship & $19(27.9)$ \\
IPE during continuing professional education & $17(25.0)$ \\
Non-IPE clinical education experiences & $15(22.1)$ \\
Workplace policies & $14(20.6)$ \\
IPE during graduate education & $9(13.2)$ \\
Other & $6(8.8)$ \\
IPE during foundational education & $5(7.4)$ \\
\hline
\end{tabular}

\section{Measurement Agreement}

An agreement was noted between the TSS and SACS assessment tools when used in this study population with an ICC $=0.43$ $(95 \% \mathrm{Cl}=0.199,0.607)$. The Bland Altman analysis also showed that SACS demonstrated appropriateness in measuring collaboration in healthcare professionals (Figure 5). 


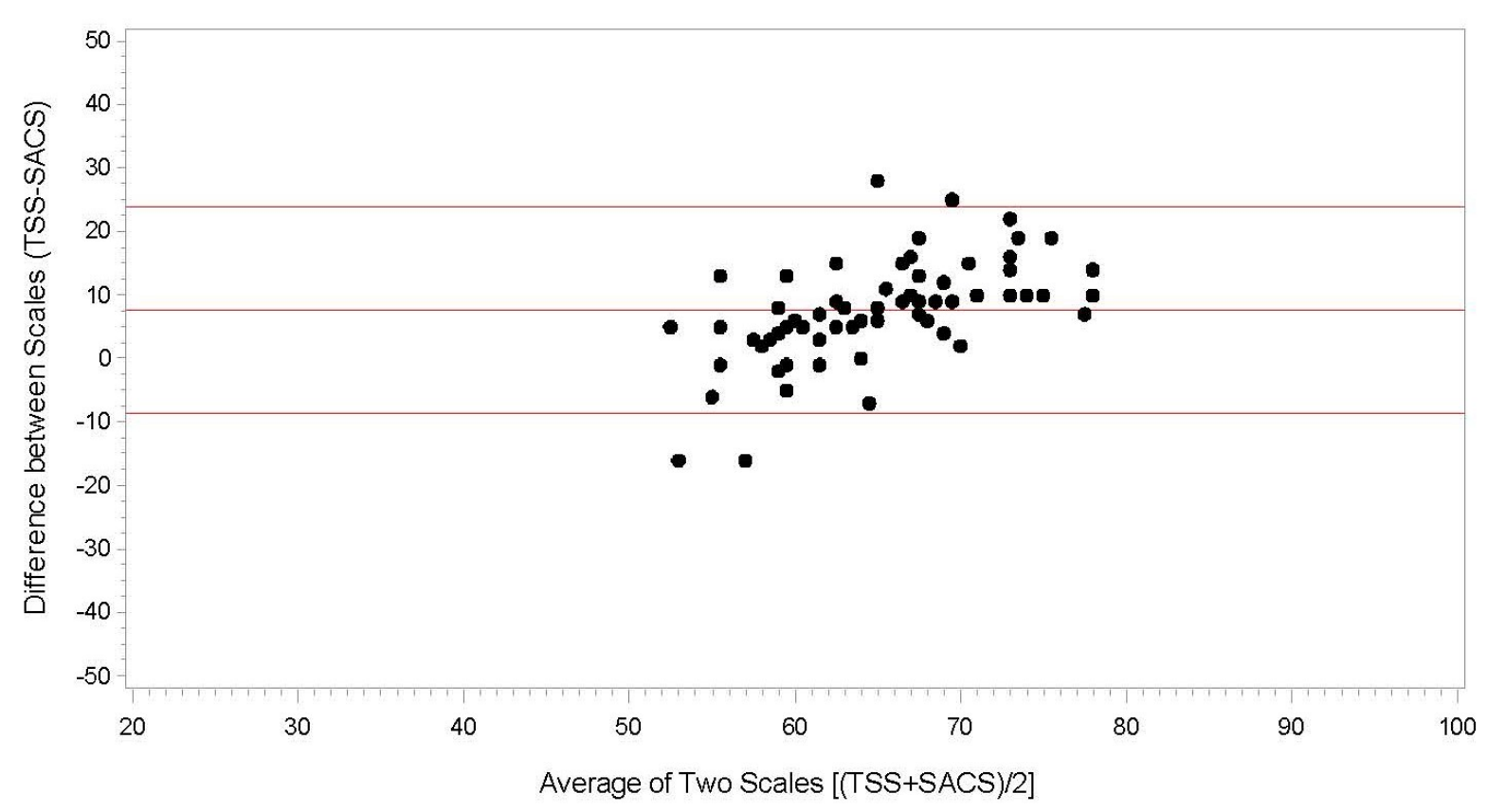

Figure 5. Bland Altman analysis of difference between TSS and SACS scores

\section{DISCUSSION}

This study is among the first to explore the distal impact of IPE on team skills and collaboration in professional practice. In this sample of healthcare professionals working on IPCP teams, exposure to IPE, timing of initial IPE exposure, and number of IPE exposures did not significantly relate to team skills or collaboration. The lack of statistically significant associations between prior IPE experience and team skills and collaboration may be the consequence of using a broad, overarching definition of IPE as an intervention. Structured IPE activities are vastly disparate across programs and institutions, ranging from small activities that may incorporate only two professions or may be point-in-time activities through broad, longitudinal curricular experiences. The content of these activities can also vary dramatically. It is not only possible, but highly likely, that these differing interventions emphasize different IPCP and collaborative skills. The assumption that all IPE interventions produce the same outcome is unfounded given their disparate approaches and emphasis. Given the difference in types and quantity of IPE experiences, our findings highlight the need for IPE effectiveness research to focus on specific interventions rather than a broad definition of IPE as any content in which "two or more professionals learn about, with, and from" each other in an explicit educational context. It is important to note that this is not an assertion that some IPE interventions are ineffective, rather an assertion that all IPE interventions may not be created equal and, thus, should be assessed independently based on their characteristics.

\section{IPE Exposure}

Trends in team skills and collaboration suggest that any exposure to IPE may improve an individual's ability to demonstrate these behaviors. IPE experiences can vary drastically across the learning continuum from classroom group work to case-based simulation to facilitated team meetings. The diversity in these experiences may prevent finding significant relationships between and IPE exposure and IPCP outcomes by reducing the effect size or creating additional statistical noise.

\section{Timing of Initial IPE Exposures}

While any exposure to IPE seems to lend towards better team skills and collaboration, the timing of initial IPE exposure may be important. Although not significant, trends in both outcome measures suggest that early exposure to IPE during foundational education may be important in optimizing team skills and collaboration in future practice. While timing of IPE exposure is often mentioned in discussions surrounding the optimal initiation of IPE curriculum and/or activities, no research currently exists exploring or documenting the optimal time for initiating IPE activities. ${ }^{33}$ It is likely that different IPE activities are more or less effective dependent upon the stage of learner. Recent research suggests that role stereotypes are already present in pre-health learners, ${ }^{34}$ thus laying the foundation for working effectively in teams before professional socialization during graduate training could by key in professional role clarity and interprofessional practice. 


\section{Number of IPE Exposures}

Across the learning continuum, the cumulative effect of IPE exposures trends towards increased levels of team skills and collaboration. Participants with two or more IPE exposures had higher TSS and SACS scores than those with one or no IPE exposures, though results were not statistically significant. As with timing of IPE exposure, the lack of significant differences is likely attributed to the diversity of IPE experiences. Of note, there is a reasonably large difference between the mean TSS scores for those with two or more experiences (TSS $M=71.4, S D=9.8$ ) and those with no IPE experience (TSS M = 66.7, SD = 9.6). The lack of statistical significance means that we cannot conclude this difference in scores is not due to chance; however, given the size of the difference, future exploration into this association is warranted. This study provides some indication that there is a possibility of a dose response relationship between IPE exposure and team skills and collaboration outcomes. Future research assessing timing and duration of IPE exposures should attempt to prospectively evaluate the long-term impact of specific IPE interventions to tease out the relationship between the amount of IPE exposure and team skills and collaborative outcomes.

\section{Contributing Factors}

Participants also indicated they believed a variety of factors contributed to their development of team skills and collaboration. The most commonly selected factor was individual personal characteristics, suggesting that there may be important, innate attributes that these healthcare professionals possess. These characteristics may lead professionals to self-select into this type of work environment that supports their collaborative nature. Previous work experience was also frequently selected as a contributing factor, suggesting that real-world socialization is important to professional development. Additionally, participants supported the IOM Framework's inclusion of workplace culture and leadership support as important contributing factors. ${ }^{16}$

\section{Assessment Tool}

This study was the first to utilize the SACS assessment tool in a sample of healthcare professionals. This tool was first tested in early learners of multiple professions and suggested to be appropriate for use with professional healthcare teams, regardless of setting and patient population. ${ }^{31}$ The TSS was originally developed for assessment of geriatric patient care teams. Although it is psychometrically strong, it must be modified to account for different patient populations. These two assessment tools are among the few that attempt to measure self-reported skills and behaviors, as compared to attitudes and beliefs. While they do measure different constructs, there is conceptual overlap between team skills and collaboration. Using both outcome measures allowed us to determine the appropriateness of using SACS in a sample of healthcare professionals. Agreement between the two measures was good, suggesting this tool may be an appropriate outcome measure for interprofessional healthcare teams.

\section{Limitations}

Limitations in this study include a small sample size made up of a convenience sample that was homogenous in outcome scores. While convenience sampling enabled access to a pool of health care professionals for surveying, biases were introduced, particularly with utilizing the Clinical Site Coordinator to help with survey distribution. Using the Clinical Site Coordinator further limited our ability to be entirely confident in the number of survey's distributed for response rate calculations. We also designed the study under the assumption that there were sufficient enabling factors in place at each of the IPCP clinics for effective teambased care. While this assumption potentially introduces uncontrolled confounding variables, we were confident that each clinic was a good model of IPCP after a tenure of participation in out institution's IPE clinical observation rotations. The cross-sectional design does not allow for true longitudinal assessment of the effect of prior IPE experiences on the development of team skills and collaboration. Participants were grouped based on their responses to questions, resulting in self-selection bias, in addition to the challenges attributed to self-rated tools. Although care was taken in crafting the definitions of IPE both broadly and at each timepoint in the learning continuum, these definitions are open to misinterpretation.

Using a catch-all definition of IPE as an intervention may be an inappropriate focus of research on its effectiveness as a pedagogical method in the development of professional skills. More emphasis should be placed on studying these same outcomes after specific IPE interventions and the nuances that make them more or less effective. Although the sample size was too small to allow for univariate comparisons across profession, descriptive statistics of the sample show differences in both TSS and SACS scores, suggesting that stratifying outcomes across professions may be an important consideration. This would enable IPE educators to make evidence-based recommendations regarding the most appropriate learning interventions for specific group compositions of professionals.

\section{CONCLUSION}

IPE as a broad definition is not sufficient to detect significant relationships with healthcare professional's team skills or collaboration. Future research should focus on similar outcomes after specific IPE interventions to determine effectiveness. Additionally, outcomes could be stratified across professions to enable specific recommendations for particular group compositions. Skill and behavior-based outcomes should be measured longitudinally, across the learning continuum, to determine true causal relationships 
between a specific IPE intervention and the development of desired skills and behaviors. Analyses accounting for other possible contributing factors, including personal characteristics, other work experiences, and workplace culture would be of great value to advance this field. Finally, the SACS assessment tool can be considered as an appropriate outcome measure of healthcare professionals' collaboration skills.

\section{REFERENCES}

1. Buttorff C, Ruder T, Bauman M. Multiple Chronic Conditions in the United States. RAND Corporation, 2017. https://www.rand.org/pubs/tools/TL221.html

2. Rosen MA, DiazGranados D, Dietz AS, et al. Teamwork in healthcare: Key discoveries enabling safer, high-quality care. Am Psychol. May-Jun 2018;73(4):433-450. [PMID 29792459]

3. World Health Organization. Framework for Action on Interprofessional Education and Collaborative Practice. Geneva, Switzerland: World Health Organization; 2010. http://www.who.int/hrh/resources/framework_action/en/.

4. Lemieux-Charles L, McGuire WL. What do we know about health care team effectiveness? A review of the literature. Medical care research and review : MCRR. Jun 2006;63(3):263-300. [PMID 16651394]

5. Hughes SL, Cummings J, Weaver F, Manheim L, Braun B, Conrad K. A randomized trial of the cost effectiveness of VA hospital-based home care for the terminally ill. Health Serv Res. Feb 1992;26(6):801-17. [PMID 1737710]

6. Jansson A, Isacsson A, Lindholm LH. Organization of health care teams and the population's contacts with primary care. Scand J Prim Health Care. Dec 1992;10(4):257-65. [PMID 1480864]

7. Naylor CJ, Griffiths RD, Fernandez RS. Does a multidisciplinary total parenteral nutrition team improve patient outcomes? A systematic review. J Parenter Enteral Nutr. Jul-Aug 2004;28(4):251-8. [PMID 15291407]

8. McAlister FA, Stewart S, Ferrua S, McMurray JJ. Multidisciplinary strategies for the management of heart failure patients at high risk for admission: a systematic review of randomized trials. Journal of the American College of Cardiology. Aug 18 2004;44(4):810-9. [PMID 15312864]

9. Holland R, Battersby J, Harvey I, Lenaghan E, Smith J, Hay L. Systematic review of multidisciplinary interventions in heart failure. Heart (British Cardiac Society). Jul 2005;91(7):899-906. [PMID 15958358]

10. Mickan SM. Evaluating the effectiveness of health care teams. Australian health review: a publication of the Australian Hospital Association. May 2005;29(2):211-7. [PMID 15865572]

11. Oandasan I, Baker G, Barker K, et al. Teamwork in Healthcare: Promoting Effective Teamwork in Healthcare in Canada. Canadian Health Services Research Foundation. 2006.

12. Morey JC, Simon R, Jay GD, et al. Error reduction and performance improvement in the emergency department through formal teamwork training: evaluation results of the MedTeams project. Health Serv Res. Dec 2002;37(6):155381. [PMID 12546286]

13. West MA, Guthrie JP, Dawson JF, Borrill CS, Carter M. Reducing patient mortality in hospitals: the role of human resource management. Journal of Organizational Behavior. 2006;27(7):983-1002. [PMID 12546286]

14. Yeatts DE, Seward RR. Reducing turnover and improving health care in nursing homes: the potential effects of selfmanaged work teams. The Gerontologist. Jun 2000;40(3):358-63. [PMID: 10853530]

15. Buring SM, Bhushan A, Broeseker A, et al. Interprofessional Education: Definitions, Student Competencies, and Guidelines for Implementation. Am J Pharm Educ. 2009;73(4):1-8. [PMID: 19657492]

16. Institute of Medicine (IOM). Measuring the Impact of Interprofessional Education on Collaborative Practice and Patient Outcomes. Washington, DC: The National Academies Press; 2015. [PMID 26803876]

17. Nisbet $G$, Lincoln $M$, Dunn S. Informal interprofessional learning: an untapped opportunity for learning and change within the workplace. J Interprof Care. 2013;27(6):469-475. [PMID 23789898]

18. Health Professions Accreditors Collaborative. Guidance on developing quality interprofessional education for health professions. Chicago, IL: Health Professions Accreditors Collaborative; 2019.

19. Loseke DR, Cahill SE. Actors in search of a character: Student social workers' quest for professional identity. Symbolic Interaction. 1986;9(2):245-258.

20. Hall P. Interprofessional teamwork: professional cultures as barriers. Journal of Interprofessional Care. 2005;19:188196. [PMID 16096155]

21. Mitchell PM, Wynia R, Golden B, et al. Core principles \& values of effective team-based health care. NAM Perspectives. Discussion Paper, Washington, DC: National Academy of Medicine; 2012. 
22. Cox M, Cuff $P, B$ randt $B$, Reeves $S$, Zierler B. Measuring the impact of interprofessional education on collaborative practice and patient outcomes. Journal of Interprofessional Care. 2016;30(1):1-3. [PMID: 26833103]

23. Kirkpatrick DL. Evaluation of Training. In: Bittel RLCLR, ed. Training and Development Handbook. McGraw-Hill; 1967:87-112.

24. Reeves S, Perrier L, Goldman J, Freeth D, Zwarenstein M. Interprofessional education: effects on professional practice and healthcare outcomes (update). Cochrane Database Syst Rev. Mar 28 2013;(3):Cd002213. [PMID 23543515]

25. Curran $\mathrm{V}$, Heath $\mathrm{O}$, Adey $\mathrm{T}$, et al. An approach to integrating interprofessional education in collaborative mental health care. Acad Psychiatry. Mar 1 2012;36(2):91-5. [PMID 22532196]

26. Darlow $B$, Brown $M$, Gallagher $P$, et al. Longitudinal impact of interprofessional education on attitudes, skills and career trajectories: a protocol for a quasi-experimental study in New Zealand. BMJ open. Jan 21 2018;8(1):e018510. [PMID 29358432]

27. Fulmer T, Hyer K, Flaherty E, et al. Geriatric interdisciplinary team training program: evaluation results. J Aging Health. 2005;17(4):443-470. [PMID: 16020574]

28. Hepburn K, Tsukuda RA, Fasser C, et al. Team skill scale. In: Heinemann GD, Zeiss AM, eds. Team Performance in Health Care: Assessment and Development. Kluwer Academic/Plenum Publishers; 2002.

29. Hyer K, Fairchild S, Abraham I, Mezey MM, Fulmer T. Unpublished factor analysis. 2000;

30. Interprofessional Education Collaborative. Core Competencies for Interprofessional Collaborative Practice: 2016 Update. Washinton, DC, Interprofessional Education Collaborative; 2016.

31. Hinyard L, Toomey E, Eliot K, Breitbach A. Student Perceptions of Collaboration Skills in an Interprofessional Context: Development and Initial Validation of the Self-Assessed Collaboration Skills Instrument. Evaluation \& the health professions. 2019 Dec;42(4):450-472 [PMID: 29334770]

32. Shrout PE, Fleiss JL. Intraclass correlations: Uses in assessing rater reliability. Psychological Bulletin. 1979;86(2):420428. [PMID 18839484]

33. Curran VR, Sharpe D, Flynn K, Button P. A longitudinal study of the effect of an interprofessional education curriculum on student satisfaction and attitudes towards interprofessional teamwork and education. Journal of Interprofessional Care. 2010/01/01 2010;24(1):41-52. [PMID 19705318]

34. Patel Gunaldo T, Augustus-Wallace A, Brisolara KF, et al. Improving stereotypes: The impact of interprofessional education in pre-health students. Journal of Interprofessional Care. 2020:1-5. [PMID: 32838602] 